\title{
An evaluation of response allocations to concurrently available slot machine simulations
}

\author{
MARK R. DIXON \\ Southern Illinois University, Carbondale, Illinois \\ and \\ OTTO H. MACLIN and DUSTIN DAUGHERTY \\ University of Northern Iowa, Cedar Falls, Iowa
}

\begin{abstract}
Gambling behavior often involves making choices between concurrently available game options. With regard to slot machine gambling, multiple games allow for choices to be made with respect to which game to play. The authors of the present study used a slot machine simulation created in Visual Basic.NET to examine gambling preference. The specific question addressed was whether gamblers prefer a smaller payout more often (averaging around every 10 spins) over a larger payout less often (averaging around every 50 spins). Two types of reinforcement were examined during the study: frequency of payouts and the amount of credits won. The results indicate that participants had a preference for a slot machine with the more frequent payout as opposed to one with more intermittent payout. These results support existing literature on theories of choice and provide a step toward understanding human gambling behavior.
\end{abstract}

The responding of an organism often occurs at the expense of missing an opportunity to engage in another behavior. Consider, for example, a slot machine player who decides to play one slot machine instead of another. This preference is initially arbitrary, and over time it may prove to be the function of outcomes obtained on that specific slot machine, superstitions that the player develops, or a combination of both. In a sense, the choice to play on one machine instead of another initially appears to be random. Yet psychological research on choice behavior, or why people choose the things they do, can serve as an initial foundation for why such a random choice may not really be random at all.

If one considers the most simple choice a slot machine player makes of playing on slot A over slot B, there are two options, A and B, and these options can be conceptualized as opportunities to win or lose various amounts of money. The programming of slot machines is such that each response is independent of the next, and no current response can predict a future response (see MacLin, Dixon, \& Hayes, 1999). This arrangement of two slot machines and their various payouts can be conceptualized as a concurrent schedule of reinforcement. Here, two options for reinforcement are available concurrently, and choosing one option results in the ability to obtain reinforcement from that option, at the expense of not obtaining reinforcement from the other option.

We thank the students in our respective labs for their help with this research. We thank the individuals who reviewed this manuscript and provided valuable feedback. Correspondence concerning this article should be addressed to O. H. MacLin, Department of Psychology, University of Northern Iowa, Cedar Falls, IA 50614 (e-mail: otto.maclin@uni.edu).
Casino gambling can be conceptualized as an activity where the individual is attempting to maximize the value derived from the experience, or the total amount of reinforcement derived from the activity. Casinos have many potentially reinforcing properties, depending on the individual. One could create a hierarchy of behaviors, such as looking at the attractive wait staff, listening to stage music, watching a performance, walking around the casino floor, drinking free or cheap drinks, eating free or inexpensive meals, watching sporting events, and then, of course, gambling. In sum, given these many potentially reinforcing behaviors, it is difficult, on an individual basis, to determine which behavior or combination of behaviors will maximize a patron's overall reinforcement. If we assume that all these variables are relatively constant across various opportunities to gamble, it appears logical to start with one variable that clearly does sustain the participation in gambling activity, the payoff of the game itself. Here it can be predicted that a patron will attempt to maximize the overall reinforcement from the situation on the basis of the value of any combination of reinforcements.

Hurlburt, Knapp, and Knowles (1980) used concurrently available online teletype computer terminals to allow participants access to a slot machine simulation that either paid off after an average number of spins, or paid off randomly. Hurlburt et al. found little difference between these two options; participants did not demonstrate differential behavior for either simulation. Participants did show, however, a preference for the machine that paid off more frequently during the training session. In the research presented here, we attempted to further examine Hurlburt et al.'s findings by having participants respond to concurrently available computerized slot machine simu- 
lations developed by the authors (MacLin et al., 1999). The present simulation is more realistic than the teletype simulation used by Hurlburt et al., in that it is configured to look and sound like the computerized slot machines that are used for Internet gambling. Also, as in a casino, both slot machine simulations in the present study were presented concurrently, side by side, on a dual-monitor display (Dixon, MacLin, \& Daugherty, 2006).

The purpose of the present study was to examine whether participants would exhibit preference for a smaller payout more often (average spins required $=10$, paying 10 points per win) over a larger payout less often (average spins required $=50$, paying 50 points per win). The formula for the matching law was used to evaluate preference. It was hypothesized that the participants would prefer the 10 -spin option because they would encounter the positive outcomes of this option more often and could adjust their betting strategies accordingly. This prediction was supported by research demonstrating that humans prefer more frequent wins (Griffiths, 1999) and that humans prefer more frequent wins during training trials (Hurlburt et al., 1980). Therefore, a slot machine that yields a smaller payout interval should be preferred over a slot machine with a larger interval, even if the overall payouts are the same, because the individual will be reinforced more frequently.

\section{Software Overview}

The slot machine simulation was written in Visual Basic.NET 2002 for any IBM-compatible computer running the Microsoft Windows XP operating system. Visual Basic.NET is a graphically oriented software program that is part of Microsoft's Developers Suite Visual Studio.NET. Although the .NET software platform is significantly more cumbersome than machine language or the $\mathrm{C}$ programming language, .NET makes up for these difficulties through its ease of accessing the Windows file libraries that make it possible to incorporate Media Player and file sharing accessibility (Dixon \& MacLin, 2003). The previous versions of slot machine simulators described by MacLin et al. (1999) and MacLin, Dixon, Robinson, and Daugherty (2006) allowed use of the software without the purchase of Visual Basic software itself. The present simulator described in this program does in fact require Visual Basic.NET, but with the steadily declining cost of the software (approximate retail is $\$ 100$ ), many researchers will find the price quite modest, given the potential.

The experimenter can control various aspects of the software simulator through a graphical interface that allows prespecification of the percentage of trials that will be wins and losses. Using drop down menus, the experimenter can also select the amount of credits that will be won by the participant on any given trial, as well as the amount of credits that each player will start with. To ensure experimental control for response bias toward the "left" or "right" sided slot machine, each machine can be randomized across trials or color coded. Finally, the experimenter can specify the number of trials required and the number of optional trials that the player will be exposed to for a given experimental session.

\section{Method}

Participants. Eighteen undergraduate students from the University of Northern Iowa were recruited through the Psychology Study Participant Manager, the Psychology Department's participant recruitment Web site. The participants were given credits toward requirements for an undergraduate psychology course.

Apparatus. The program is based on a version of a slot machine created in Visual Basic.NET by one of the authors (MacLin et al., 1999). The program has been modified to run on a computer supporting dual monitors (see Figure 1) and to accept graphics from Internet-based gambling sites (Dixon \& Schreiber, 2004). For example, sounds and graphics can be downloaded and applied as "skins" to the existing program. In addition, graphics of Internet banners and advertisements can be displayed to provide further realism. The reels can be reconfigured by editing the images onto a single graphic representing a reel. The program was modified to permit use of an Excel spreadsheet to pass values such as the reel combination and payout amount to the Visual Basic program. Complete code for running a similar program with a single monitor can be found in MacLin et al. (2006).

The slot machines, which were based on an Internet slot machine called Double Magic (n.d.), included many of the features of an actual slot machine (www.gambling-slots .info/slots/double_magic.htm) (see Figure 2). The reel was modified to include six possible symbols that could be displayed at any one time: a cherry, a plum, a bell, a 1000 symbol, a "bar," and an orange, with blank spaces between symbols. Winning combinations were displayed on the right of the reels in a payoff table. There were three buttons on the machine that the participants could manipulate by placing the cursor over the button and clicking with the mouse: "bet one," "bet max," and "spin." The "spin" button was used when the individual was ready to spin the

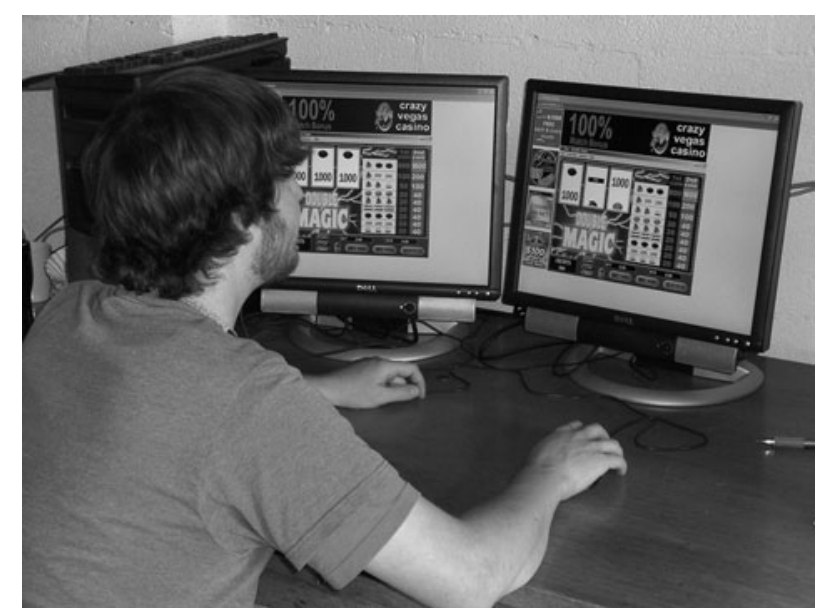

Figure 1. Participant playing the dual-monitor slot machines. 


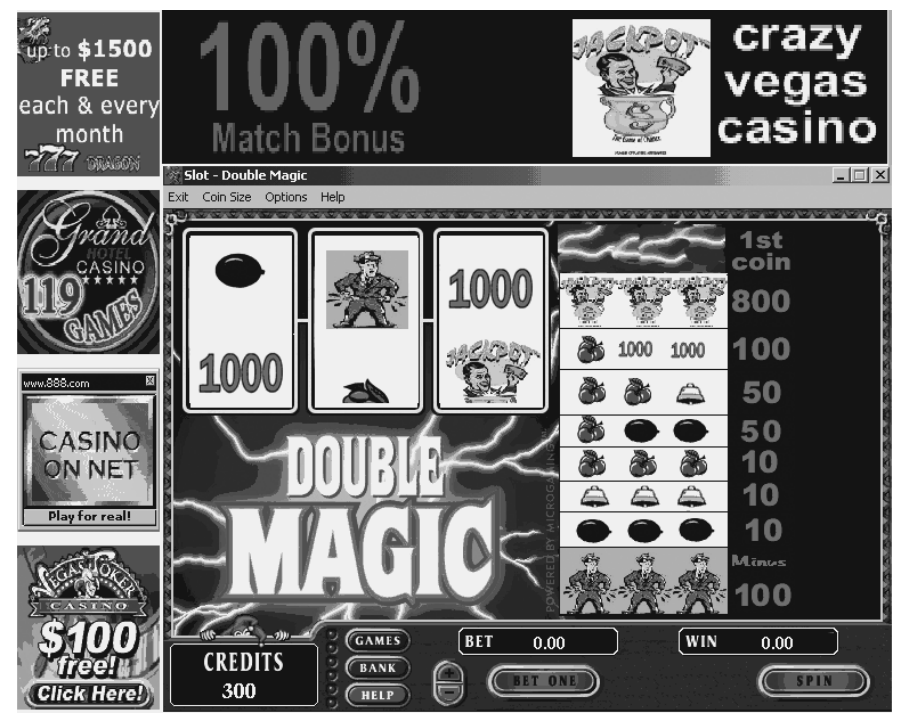

Figure 2. Screen print of the Double Magic graphics based on the actual Internet gambling site.

reels, but it could not be operated until the participant had bet at least one credit. If the participant bet two credits, by clicking on the "bet max" button or clicking the "bet one" button twice, the payout for a winning combination was doubled. Both slot machines could not be played at the same time. Thus a changeover delay occurred, consisting of a pausing of the program for a few seconds before allowing the participant to switch to the other option and click on the bet button, and the duration of the spin.

Sounds were played following different behaviors (after a button was clicked, during a spin, and following a win), to create a more realistic casino environment. The slot machines displayed three different text boxes at the bottom of the machine, which gave the total credits, the amount bet, and the amount won for the recent spin. The program recorded which machine was being played and the total credits remaining for each machine.

Because the program was used to examine preference, it was important for the participants to play both machines during training. A control panel added to the program included an option to enable the researcher to "lock out" the participant from playing one of the slot machines for a given amount of time, to effectively force the participant to familiarize himself or herself with each slot machine. After the initial lockout period of $5 \mathrm{~min}$, the participant was allowed to move freely between the two slots by moving the mouse cursor from one monitor to the other. An option was added to the control panel to determine which slot was presented on the left or the right side of the participant. This allows researchers to control for any potential side preference that might confound actual game preference.

Each machine was programmed to run for 300 trials, so a participant showing equal preference for both machines would run 600 trials, given enough time. One slot machine was programmed to pay out 10 credits per win after an average of 10 spins, whereas the other slot machine was programmed to pay out 50 credits per win after an average of 50 spins. With these parameters, both slot machines would pay out the equivalent amount across every 300 trials, if one assumes that equal bet amounts were placed for each machine.

Procedure. Participants were randomly assigned to one of four conditions (see Table 1). In Condition 1, the payout 10 slot machine was presented first and on the left monitor, whereas in Condition 2, the payout 10 slot machine was presented first but on the right monitor. In Condition 3, the payout 10 slot machine was presented second and on the right side, whereas in Condition 4, it was presented second, but on the right side. The participants were informed that they would be playing two simulated slot machines, each starting with 300 credits. Participants were informed that their names would be entered into a drawing for a $\$ 25$ gift certificate for every 10 credits won over the initial 600 credits (300 on each machine). After participants read and signed informed consents, they were directed to a computer where they read a set of instructions, which included directions for how to play the slot machine and information regarding the training trialsspecifically, that the participant would be locked out of a slot machine for a given amount of time. Participants then proceeded to interact with the slot machine for $50 \mathrm{~min}$, after which they were debriefed and allowed to leave the laboratory.

Table 1

Slot Machine Condition: Position and Order of the Payout 10 Option

\begin{tabular}{ccc}
\multicolumn{3}{c}{ Order of the Payout 10 Option } \\
\hline \multirow{2}{*}{ Order } & Left Side & Right Side \\
\cline { 2 - 3 } Shown first & Condition 1 & Condition 2 \\
Shown second & Condition 3 & Condition 4 \\
\hline
\end{tabular}




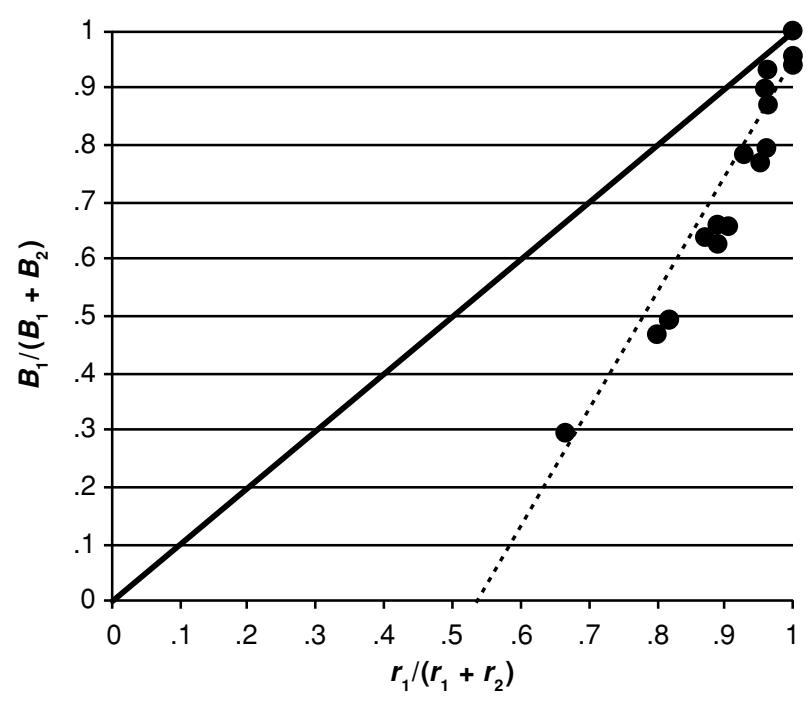

Figure 3. $B_{1}$ and $B_{2}$ represent the total number of times that the participant clicked on the button to spin the payout 10 or payout 50 slot machine, respectively. The $y$-axis thus represents the ratio of responses on the payout 10 slot machine over the total responses made on both slot machines during the experiment. The notation $r_{1}$ refers to the frequency of payouts with the payout 10 slot machine, whereas $r_{2}$ refers to the frequency of payouts with the payout 50 slot machine. The $x$-axis thus represents the ratio of payouts obtained from the payout 10 over the total payouts from both slot machines. The solid black line through the diagonal of Figure 1 represents a ratio predicting that response allocations would be proportional to obtained reinforcers, whereas the dotted black line represents a regression line fitted to the least sum of squares, $y=x * .998+.54 ; r(17)=540.87, p<.001 ; S E=.029$.

\section{Results}

Order and side preference. Independent $t$ tests were run to determine whether preferences existed for side and lockout sequence. There were no significant differences for side $[t(1,16)=.579$, n.s. $]$ or for lockout sequence $[t(1,16)=.308$, n.s. $]$. Therefore the data were collapsed across these factors for all subsequent analyses.

Preference. In Figure 3, any data point above .5 on the $y$-axis indicates that the individual demonstrated a preference for playing the payout 10 slot machine, whereas points below .5 demonstrate a preference for playing the payout 50 slot machine. Likewise, any point beyond .5 on the $x$-axis demonstrates that there were more payouts with the payout 10 slot machine than with the payout 50 slot machine. Participants 9, 12, and 14 show ratio scores of $(1,1)$ which indicate an absolute preference for the payout 10; no payouts were received for the payout 50 . Participant 6 shows a ratio score of $(.67, .29)$, indicating a preference for the payout 50, although the payout 10 produced more overall payouts. Data points from all but 3 participants are located in the upper right quadrant of Figure 3. This indicates that $83 \%$ of the participants preferred the payout 10 slot machine, though $100 \%$ of them were reinforced more often with payouts on the variable ratio 10 schedule.

Total credits. Credits refer to the number of points that an individual has obtained on a particular machine, and total credits are the number of cumulative points that a participant has obtained on both machines, including the initial 600 credits that the participants were given at the beginning of the study. Total credits are independent of the number of payouts, in that the participant could vary the amount of the bet. The mean number of total credits a participant obtained at the end of the study was 622 , with a range of 557 to 711 . Figure 4 illustrates the relationship between responding ratios and credits ratios and indicates a preference toward the payout 10 slot machine. This preference could have been the result of more frequent credit accumulations of the payout 10 option, or perhaps of the training trials' extending into the experimental trials. In

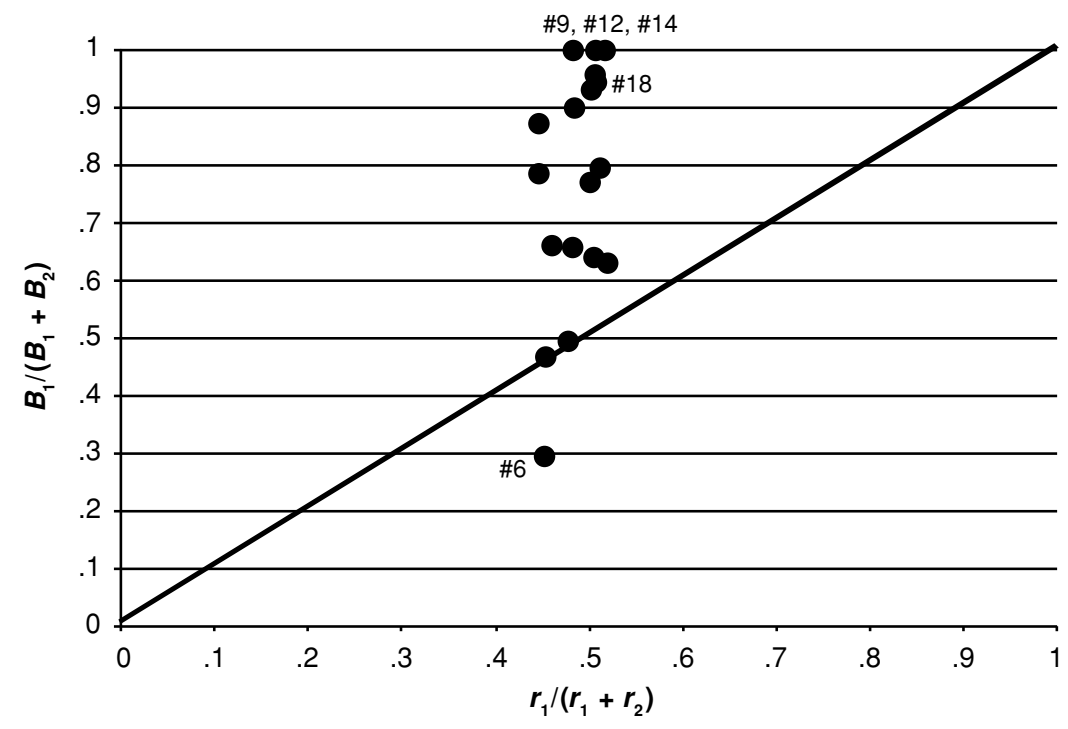

Figure 4. The same metrics as in Figure 3, here with credits earned as the dependent variable. 
other words, the participants may have already emitted a number of responses in the training trials, which then were carried over into the experimental trials, so that fewer spins were required for reinforcement.

\section{Discussion}

As was predicted, the present study shows that most of the participants preferred a slot machine option that produced small yet more frequent payouts over an option producing larger yet less frequent payouts. These findings support earlier predictions suggesting that participants prefer more frequent wins (Griffiths, 1999) and prefer slots that pay out more frequently in training sessions (Hurlburt et al., 1980). However, the results of these previous studies were limited, because one could not differentiate whether more frequent wins overall or more frequent wins during the training trials were controlling the behavior. Preference in the present study, however, was demonstrated with the use of realistic slot machine computer simulations displayed concurrently on separate monitors controlled by the same computer (Dixon et al., 2006): Participants clearly preferred more frequent, low payouts over less frequent, higher payouts.

This is the first study to examine concurrent slot machine preferences when two machines are available simultaneously and a choice for one decreases the opportunity for choices on the other. It is our hope that the experimental preparation of a dual-monitored simulated slot machine will be replicated by other researchers interested in examining gambling behavior. Using the dual-monitor system with the software described by MacLin et al. (1999), one can easily manipulate other factors present in a casino environment, such as display types, sounds from the slot machine, and number of payoff lines. Future research might also examine some of the important components within the slot machine simulation itself, such as the near miss (Dixon \& Schreiber, 2004), auditory feedback, and the perceived ability to exert control over the slot machine outcome. If we can predict responding on slot machines of recreational gamblers, we might be on the road to better understanding the variables that contribute to excessive, pathological gambling. The present data suggest that like many other forms of behavior, gambling can lend itself to order and predictability.

\section{REFERENCES}

Dixon, M. A., \& MacLin, O. H. (2003). Visual Basic for behavioral psychologists. Reno, NV: Context Press.

DiXon, M. R., MacLin, O. H., \& Daugherty, D. (2006). Dual-monitor preference paradigm: A new method for examining preference. Manuscript submitted for publication.

Dixon, M. R., \& Schreiber, J. E. (2004). Near-miss effects on response latencies and win estimations of slot machine play. Psychological Record, 54, 335-348.

Double Magic Slot Machine (n.d.). Retrieved August 20, 2002, from www.gambling-slots.info/slots/double_magic.htm.

Griffiths, M. (1999). Gambling technologies: Prospects for problem gambling. Journal of Gambling Studies, 15, 265-283.

Hurlburt, R. T., Knapp, T. J., \& Knowles, S. H. (1980). Simulated slot-machine play with concurrent variable ratio and random ratio schedules of reinforcement. Psychological Records, 47, 635-639.

MacLin, O. H., Dixon, M. R., \& HaYes, L. J. (1999). A computerized slot machine simulation to investigate the variables involved in gambling behavior. Behavior Research Methods, Instruments, \& Computers, 31, 731-734.

MacLin, O. H., Dixon, M. R., Robinson, A., \& Daugherty, D. (2006). Writing a simple slot machine simulation program. In P. M. Ghezzi, C. Lyons, M. Dixon, \& G. Wilson (Eds.), Gambling: Behavior theory, research, and application (pp. 127-154). Reno, NV: Context Press.

(Manuscript received March 14, 2006; revision accepted for publication May 8, 2006.) 